\title{
Hypotension caused by oral administration of 5-aminolevulinic acid persists after surgery in patients undergoing transurethral resection of bladder tumor under spinal anesthesia
}

\author{
Masami Sato*, Tsutomu Yanagisawa, Sonoko Minamino and Toshiyuki Arai
}

To the Editor,

5-Aminolevulinic acid (ALA) is utilized for photodynamic diagnosis to detect superficial bladder cancer and its adverse effect is hypotension [1-5]. Compared to general anesthesia, spinal anesthesia is not indicated as an increased risk factor for 5-ALA-induced hypotension from anesthesia induction to the start of surgery in patients undergoing transurethral resection of bladder tumor (TUR-BT) with 5-ALA [2, 3]. However, no studies exist that have investigated the precise perioperative hypotensive effects by 5-ALA under spinal anesthesia. This retrospective study assessed the hypotensive effect of 5-ALA on hemodynamic change of spinal anesthesia in patients undergoing TUR-BT not only during surgery but also after the ward admission.

The medical records of 129 patients who underwent TUR-BT under spinal anesthesia using 0.5\% hyperbaric bupivacaine in Kyoto City Hospital between January 2018 and March 2019 were divided into two groups, based on whether 5-ALA was used (ALA group, $n=66$ ) or not used (control group, $n=63$ ). The ALA group received 5-ALA $(20 \mathrm{mg} / \mathrm{kg})$ orally $3 \mathrm{~h}$ before the start of surgery. The change in noninvasive blood pressure (BP) on the day of surgery and the dosage of vasopressors used during spinal anesthesia were analyzed.

No significant difference existed between the two groups in the patients' preoperative characteristics. Intravenous fluid volume during anesthesia was significantly higher in the ALA group than in the control group (Table 1). Systolic and diastolic BP were significantly lower in the ALA group than in the control group from spinal anesthesia until $2 \mathrm{~h}$ after the ward admission. In the ALA group, systolic and diastolic BP significantly remained decreased from spinal anesthesia until $2 \mathrm{~h}$ after the ward admission (Fig. 1). The total dosage of ephedrine and phenylephrine were higher in the ALA group than in the control group (Table 1).

Additive hypotensive effect induced by spinal anesthesia and 5-ALA was prominent after spinal anesthesia induction not only during surgery but also for $2 \mathrm{~h}$ after the ward admission, as shown by the BP changes in our ALA group. Since the duration of the hypotensive effect by 5-ALA is longer than that of hyperbaric bupivacaine of spinal anesthesia, the systolic BP in the ALA group did not return to the baseline level even $2 \mathrm{~h}$ after the ward admission, unlike in the control group. We cannot easily state that spinal anesthesia using hyperbaric bupivacaine is a safe technique from our result, when considering the postoperative period. Our reflection point is that hypotension may be encouraged by hypovolemia in the ALA group, because all patients did not have intravenous fluids after 5-ALA administration before spinal anesthesia. Other risk factors that increase the incidence of 5-ALA-induced hypotension have been reported to be the regular use of antihypertensive drugs, particularly renin-angiotensin system inhibitor drugs $[2,4]$ and a history of ALA-induced hypotension [5].

* Correspondence: masami@kb3.so-net.ne.jp

Department of Anesthesia, Kyoto City Hospital, 1-2 Mibuhigashitakada-cho, Nakagyo-ku, Kyoto 604-8845, Japan 
Table 1 The patients' preoperative characteristics, anesthesia profiles and the drugs administered during anesthesia

\begin{tabular}{|c|c|c|c|}
\hline & Control group $(n=63)$ & ALA group $(n=66)$ & $P$ value \\
\hline Age (years) & $71 \pm 11$ & $74 \pm 10$ & 0.27 \\
\hline Height $(\mathrm{cm})$ & $162 \pm 15$ & $161 \pm 14$ & 0.58 \\
\hline Weight (kg) & $65 \pm 17$ & $65 \pm 18$ & 0.97 \\
\hline Sex (male/female) & $59(94 \%) / 4(6 \%)$ & $55(83 \%) / 11(17 \%)$ & 0.07 \\
\hline Regular use of antihypertensive drug & $24(38 \%)$ & $23(35 \%)$ & 0.70 \\
\hline Regular use of renin-angiotensin system inhibitor & $14(22 \%)$ & $11(17 \%)$ & 0.42 \\
\hline Duration of surgery (minutes) & $43 \pm 25$ & $44 \pm 20$ & 0.84 \\
\hline Duration of anesthesia (minutes) & $75 \pm 28$ & $80 \pm 22$ & 0.25 \\
\hline Dose of $0.5 \%$ hyperbaric bupivacaine of spinal anesthesia $(\mathrm{mL})$ & $3.1 \pm 0.3$ & $3.0 \pm 0.3$ & 0.20 \\
\hline Sensory block level just before surgery ${ }^{a}$ & T8 (T2-T11) & $\mathrm{T} 6(\mathrm{~T} 2-\mathrm{L} 1)$ & 0.26 \\
\hline Sensory block level at the end of surgery ${ }^{a}$ & $\mathrm{~T} 6(\mathrm{~T} 2-\mathrm{T} 12)$ & $\mathrm{T} 5(\mathrm{~T} 2-\mathrm{T} 12)$ & 0.49 \\
\hline Intravenous fluid volume during anesthesia (mL) & $570 \pm 191$ & $758 \pm 284$ & $<0.001$ \\
\hline Use of vasopressors ${ }^{\mathrm{b}}$ & $10(16 \%)$ & $47(71 \%)$ & $<0.001$ \\
\hline Use of ephedrine & $8(13 \%)$ & $37(56 \%)$ & $<0.001$ \\
\hline Total dose of ephedrine (mg) & $1.0 \pm 3.0$ & $6.9 \pm 9.0$ & $<0.001$ \\
\hline Use of phenylephrine & $6(10 \%)$ & $30(45 \%)$ & $<0.001$ \\
\hline Total dose of phenylephrine $(\mathrm{mg})$ & $0.013 \pm 0.042$ & $0.21 \pm 0.34$ & $<0.001$ \\
\hline
\end{tabular}

Values are presented as mean \pm standard deviation or number (\%)

${ }^{\mathrm{a} M e d i a n}$ (maximum-minimum)

${ }^{\mathrm{b}}$ Ephedrine or phenylephrine or both were used

ALA aminolevulinic acid
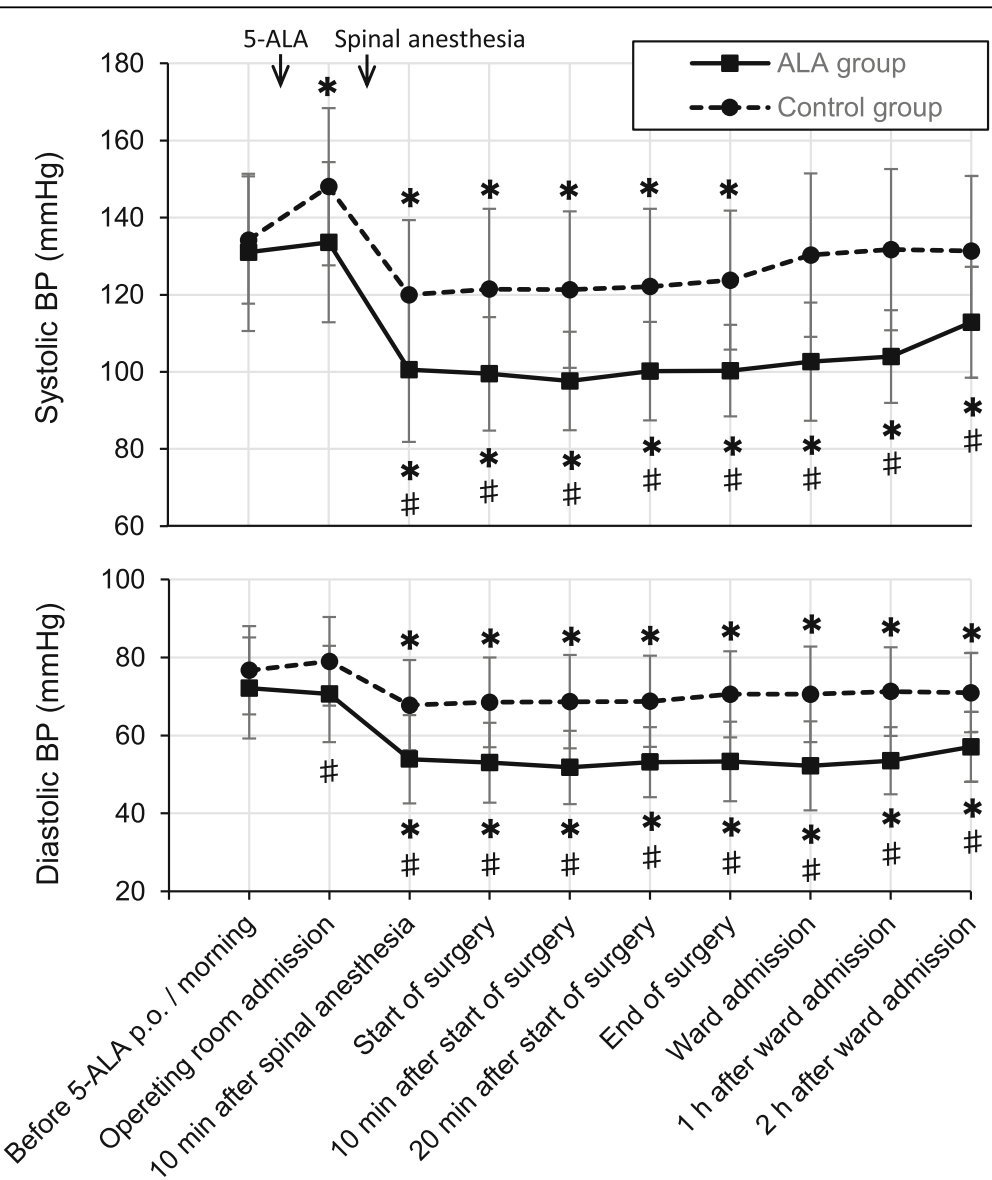

Fig. 1 Time courses of systolic blood pressure and diastolic blood pressure. Values are presented as mean \pm standard deviation. ${ }^{*} P<0.05$, post-operating room admission versus before 5-ALA p.o./morning. ${ }^{~} P<0.05$, the ALA group versus the control group. ALA aminolevulinic acid, BP blood pressure, $p .0$. per os 
In conclusion, orally administered 5-ALA before TUR-BT under spinal anesthesia induced significant hypotension after spinal anesthesia induction until $2 \mathrm{~h}$ after surgery and an increased need for vasopressors and intravenous volume. Anesthesiologists should have a strategy for safe anesthetic management against longlasting hypotension by 5-ALA.

\section{Abbreviations}

ALA: Aminolevulinic acid; TUR-BT: Transurethral resection of bladder tumor;

BP: Blood pressure

\section{Acknowledgements}

Not applicable

\section{Authors' contributions}

All authors contributed to the study conception and design. Material preparation, data collection, and analysis were performed by MS, TY, and SM. The first draft of the manuscript was written by MS and supervised by TA. All authors read and approved the final manuscript.

\section{Funding}

None

\section{Availability of data and materials}

The datasets used and analyzed during the current study are available from the corresponding author on reasonable request.

\section{Ethics approval and consent to participate}

This study was approved by the institutional ethics committee of Kyoto City Hospital (approval no. 514) and the requirement for informed consent was waived according to the retrospective nature of the study.

\section{Consent for publication}

Not applicable

\section{Competing interests}

The authors declare that they have no competing interests

Received: 5 October 2020 Revised: 2 November 2020

Accepted: 10 November 2020 Published online: 21 November 2020

\section{References}

1. Herman MA, Webber J, Fromm D, Kessel D. Hemodynamic effects of 5aminolevulinic acid in humans. J Photochem Photobiol B. 1998:43:61-5.

2. Nohara T, Kato Y, Nakano T, Nakagawa T, Iwamoto H, Yaegashi H, et al. Intraoperative hypotension caused by oral administration of 5aminolevulinic acid for photodynamic diagnosis in patients with bladder cancer. Int J Urol. 2019;26:1064-8.

3. Yatabe T, Karashima T, Kume M, Kawanishi Y, Fukuhara H, Ueba T, et al. Identification of risk factors for post-induction hypotension in patients receiving 5-aminolevulinic acid: a single-center retrospective study. JA Clin Rep. 2020;6:35.

4. Chung IW, Eljamel S. Risk factors for developing oral 5-aminolevulinic acidinduced side effects in patients undergoing fluorescence guided resection. Photodiagn Photodyn Ther. 2013;10:362-7.

5. Bondad J, Aboumarzouk OM, Moseley H, Kata SG. Oral 5-aminolevulinic acid induced photodynamic diagnostic ureterorenoscopy - does the blood pressure require monitoring? Photodiagn Photodyn Ther. 2013;10:39-41.

\section{Publisher's Note}

Springer Nature remains neutral with regard to jurisdictional claims in published maps and institutional affiliations. 